\title{
Erratum: the effective action of double field theory
}

\author{
Gerardo Aldazabal, ${ }^{a}$ Walter Baron, ${ }^{b}$ Diego Marqués ${ }^{c}$ and Carmen Núñez ${ }^{b, d}$ \\ ${ }^{a}$ Centro Atómico Bariloche, and Instituto Balseiro (CNEA-UNC) and CONICET, \\ 8400 S.C. de Bariloche, Argentina \\ ${ }^{b}$ Instituto de Astronomía y Física del Espacio (CONICET-UBA), \\ C.C. 67 - Suc. 28, 1428 Buenos Aires, Argentina \\ ${ }^{c}$ Institut de Physique Théorique, CEA/ Saclay, \\ 91191 Gif-sur-Yvette Cedex, France \\ ${ }^{d}$ Departamento de Física, FCEN, Universidad de Buenos Aires \\ Buenos Aires, Argentina \\ E-mail: aldazaba@cab.cnea.gov.ar, w_baron@iafe.uba.ar, \\ diego.marques@cea.fr, carmen@iafe.uba.ar
}

ERRATUM TO: JHEP11(2011)052

Keywords: Flux compactifications, String Duality

ARXIV EPRINT: 1109.0290

The first equation in (3.18) should read

$$
B_{\mu \nu}=\breve{B}_{\mu \nu}-\frac{1}{2}\left(\mathcal{A}^{a}{ }_{\mu} \mathcal{B}_{a \nu}-\mathcal{A}^{a}{ }_{\nu} \mathcal{B}_{a \mu}\right)-\mathcal{A}^{a}{ }_{\mu} \mathcal{A}^{a}{ }_{\nu} b_{a b} .
$$

The second and third equations in (3.22) should be replaced by

$$
\begin{aligned}
\hat{\mathcal{H}}^{A \nu}(x) & =A^{A}{ }_{\mu} g^{\mu \nu}, \\
\hat{\mathcal{H}}_{A \nu} & =-\mathcal{H}_{A B} A^{B}{ }_{\nu}-A_{A \rho} g^{\rho \mu}\left(B_{\mu \nu}+\frac{1}{2} A_{\mu}^{B} A_{B \nu}\right) .
\end{aligned}
$$

